\title{
ENTEROPARASITOSES EM ESCOLARES DO DISTRITO DE MARTINÉSIA, UBERLÂNDIA, MG: UM ESTUDO-PILOTO
}

\author{
Carla Borges Ferreira e Oswaldo Marçal Junior
}

\begin{abstract}
Este trabalho avalia a ocorrência de parasitas intestinais em estudantes do Distrito de Martinésia, município de Uberlândia (MG). Foram examinadas 103 crianças, no periodo de setembro a novembro de 1995, segundo o método de Lutz ou Hoffman, Pons E Janer. O coeficiente geral de prevalência foi de 22,3\% e os índices de infecção mais elevados foram observados no grupo etário 8 a 9 anos (34,8\%), nos moradores da vila $(30,0 \%)$ e no sexo feminino (26,9\%). Helmintoses e protozooses apresentaram taxas de prevalência similares (10,7\% e 12,6\%, respectivamente). Giardia lamblia foi o único protozoário parasito verificado e apenas um caso de poliparasitismo foi encontrado. Conclui-se que a prevalência de enteroparasitoses no grupo estudado é menor do que o esperado para uma comunidade rural, o que é, provavelmente, uma conseqüência das boas condições sanitárias presentes naquele distrito.
\end{abstract}

Palavras-chaves: Enteroparasitoses. Parasitas intestinais. Prevalência.

Nas últimas décadas, têm sido observadas ligeiras reduções nas taxas globais de prevalência de diferentes infecções parasitárias, mas em contrapartida observa-se um sensível aumento do número absoluto de casos 17. Estimativas recentes indicam que somente Ascaris lumbricoides aflige cerca de $1 / 4$ da população mundial, distribuindo-se por mais de 150 países e territórios 6 . Neste contexto, as parasitoses intestinais ou enteroparasitoses são responsáveis por altos índices de morbidade, principalmente nos países em desenvolvimento, onde o crescimento populacional não é acompanhado da melhoria das condições de vida da população14 1719 .

No Brasil, as enteroparasitoses figuram entre os principais problemas de saúde pública2 416; e no entanto, a investigação parasitológica tem sido amplamente negligenciada no país. Em Uberlândia e região, levantamentos parasitológicos recentes têm retratado somente a situação observada nas zonas urbanas, desconsiderando a realidade das comunidades rurais 13 .

Este trabalho se constitui na etapa inicial de uma ampla investigação da transmissão de parasitas intestinais na comunidade de Martinésia,

Departamento de Biociências, Centro de Ciências Biomédicas da Universidade Federal de Uberlândia, Uberlândia, MG Endereço para correspondência: Prof. Oswaldo Marçal Jr. Dept $^{\circ}$ de Biociências/CCB/UFU, Campus Umuarama, CP 593, 38400-902 Uberlândia, MG, Brasil.

Telefax: (034) 218-2243.

Recebido para publicação em 06/12/96. importante distrito rural do município de Uberlândia, MG, e tem como objetivo avaliar os índices de prevalência das enteroparasitoses nas crianças e jovens estudantes locais, o principal grupo de risco para aquisição de infecções intestinais e aquele onde, geralmente, são observados os maiores problemas físicos, mentais e sociais associados a estas infecções 1318 .

\section{MATERIAL E MÉTODOS}

Área de estudo. Segundo dados da Prefeitura Municipal de Uberlândia (MG), o distrito de Martinésia apresenta atualmente uma população de aproximadamente 1.000 habitantes, que tem nas atividades agropecuárias sua base econômica. O distrito é formado por um pequeno núcleo urbano (vila), que possui pavimentação asfáltica, rede de esgoto e tratamento de água, além de diversas propriedades rurais (fazendas, sítios e chácaras). Um posto de saúde municipal presta atendimento médico-odontológico à população, embora não haja médicos ou dentistas permanentes no distrito. A Escola Municipal Martinésia, a única do distrito, apresentava, em 1995, um total de 145 alunos matriculados da pré-escola à $8^{\text {a }}$ série, nos períodos matutino e vespertino.

Exame de Fezes. Foram distribuídos coletores universais, contendo formol a $10 \%$ e previamente identificados com o nome, idade e série de cada criança, a todos os alunos matriculados nos turnos matutino e vespertino da escola local. Os escolares foram orientados sobre a importância do exame e receberam um 
manual, encaminhado aos pais ou responsáveis, indicando os cuidados a serem observados durante a colheita do material. Foram preparadas 2 lâminas por amostra individual (coradas em lugol), segundo o método de Lutz ${ }^{12}$, mais conhecido como método de Hoffman, Pons e Janer (HPJ)11. Todos os exames foram realizados no Laboratório de Parasitologia do Departamento de Patologia da Universidade Federal de Uberlândia (UFU) e os resultados obtidos foram devolvidos aos pais e/ou responsáveis, por intermédio da escola. Os indivíduos infectados foram encaminhados ao posto de saúde do distrito, a fim de receber tratamento.

Análise estatística. A análise estatística dos resultados foi realizada por meio do programa EPIINFO ${ }^{7}$. As diferenças observadas na distribuição dos casos segundo sexo, zona de habitação e grupo etário foram testadas ( $\left.\mathrm{X}^{2}\right)$ e o nível de significância aceito foi de $95 \%$ $(\mathrm{p}<0,05)$.

\section{RESULTADOS}

Foram examinados 103 alunos (71\% do total de alunos matriculados), dos quais $50,5 \%$ eram do sexo feminino e $61,2 \%$ residiam no campo. Os indivíduos investigados apresentaram a seguinte distribuição etária: 6-7 (18,4\%), 8-9 (22,3\%), 10-11 (21,4\%), 12-13 (13,6\%) e $\geq 14$ anos $(24,3 \%)$.

O coeficiente geral de prevalência de enteroparasitoses foi de 22,3\%, sendo registrados 11 casos de helmintoses e 13 de protozooses $(10,7 \%$ e $12,6 \%$, respectivamente). O sexo feminino apresentou prevalência de 26,9\%, contra $17,6 \%$ no sexo masculino. Os indivíduos residentes na vila apresentaram coeficiente de prevalência de $30,0 \%$, contra $17,5 \%$ nos residentes nas fazendas (Tabela 1). No grupo etário de 8-9 anos foi registrada prevalência de $34,8 \%$ (Figura 1).

\begin{tabular}{|c|c|c|c|c|c|c|}
\hline \multirow{3}{*}{ Sexo } & \multicolumn{4}{|c|}{ Local de habitação } & & \\
\hline & \multicolumn{2}{|c|}{ vila } & \multicolumn{2}{|c|}{ campo } & \multicolumn{2}{|c|}{ Total } \\
\hline & $\mathrm{n}^{\mathrm{o}}$ & $\%$ & $\mathrm{n}^{\mathrm{o}}$ & $\%$ & $\mathrm{n}^{\mathrm{o}}$ & $\%$ \\
\hline Masculino & $4 / 40$ & 10,0 & $5 / 63$ & 7,9 & $9 / 51$ & 17,6 \\
\hline Feminino & $8 / 40$ & 20,0 & $6 / 63$ & 9,5 & $14 / 52$ & 26,9 \\
\hline Total & $12 / 40$ & 30,0 & $11 / 63$ & 17,5 & $23 / 103$ & 22,3 \\
\hline
\end{tabular}

Os grupos etários com 6-7, 10-11 e 12-13 anos apresentaram as maiores prevalências para protozooses intestinais, enquanto os

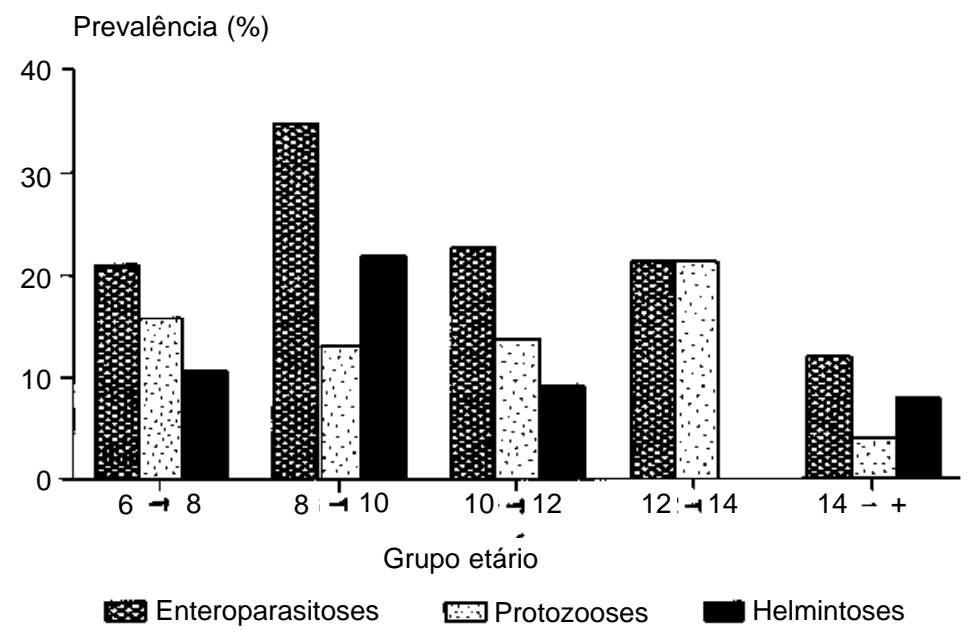

Figura 1- Coeficientes de prevalência de parasitoses intestinais, segundo grupo etário, em escolares do distrito de Martinésia, Uberlândia $(M G), 1995$. 
Ferreira CB, Marçal Junior O. Enteroparasitoses em escolares do distrito de Martinésia, Uberlândia, MG: um estudo-piloto. Revista da Sociedade Brasileira de Medicina Tropical 30:373-377, set-out, 1997.

indivíduos com 8-9, e os com idade de 14 anos ou mais apresentaram-se mais parasitados por helmintos (Figura 1). Um único caso de poliparasitismo foi registrado $(1,0 \%)$ e esta associação ocorreu entre os helmintos Hymenolepis nana, Hymenolepis diminuta, Trichuris trichiura e o protozoário Giardia lamblia.

As maiores taxas de prevalência de protozooses ocorreram no sexo feminino $(19,2 \%)$ e nos alunos residentes no distrito $(15,0 \%)$, sendo que Giardia lamblia foi a única espécie parasita encontrada. Para as helmintoses foram registradas taxas de prevalência mais elevadas no sexo masculino
(11,8\%) e novamente nos moradores do distrito (12,5\%) (Tabela 2). Os helmintos mais representativos foram os ancilostomatídeos $(3,8 \%)$. Estes parasitos estiveram presentes nos grupos etários de 8-9 e com idade de 14 anos ou mais, mostrando-se ausentes nos demais. As infecções por Strongyloides stercoralis, Hymenolepis nana e Enterobius vermicularis apresentaram coeficiente de prevalência de 1,9\%, enquanto em H. Diminuta, Ascaris lumbricoides e T. Trichiura este índice foi de $1,0 \%$, o que representa um único caso para cada parasitose (Figura 2).

Além das espécies parasitas foram encontradas também espécies comensais: Entamoeba coli,

Tabela 2 - Coeficientes de prevalência de protozooses e helmintoses, segundo sexo e local de habitação, em escolares do distrito de Martinésia, Uberlândia $(M G), 1995$

\begin{tabular}{|c|c|c|c|c|c|c|c|c|}
\hline \multirow{3}{*}{ Parasitose } & \multicolumn{4}{|c|}{ Sexo } & \multicolumn{4}{|c|}{ Local de habitação } \\
\hline & \multicolumn{2}{|c|}{ masculino } & \multicolumn{2}{|c|}{ feminino } & \multicolumn{2}{|c|}{ vila } & \multicolumn{2}{|c|}{ campo } \\
\hline & $\mathrm{n}^{\mathrm{O}}$ & $\%$ & $\mathrm{n}^{\mathrm{o}}$ & $\%$ & $\mathrm{n}^{\mathrm{o}}$ & $\%$ & $\mathrm{n}^{\mathrm{o}}$ & $\%$ \\
\hline Protozoose & $3 / 51$ & 5,9 & $10 / 52$ & 19,2 & $6 / 40$ & 15,0 & $7 / 63$ & 11,1 \\
\hline Helmintose & $6 / 51$ & 11,8 & $5 / 52$ & 9,6 & $5 / 40$ & 12,5 & $6 / 63$ & 9,5 \\
\hline
\end{tabular}

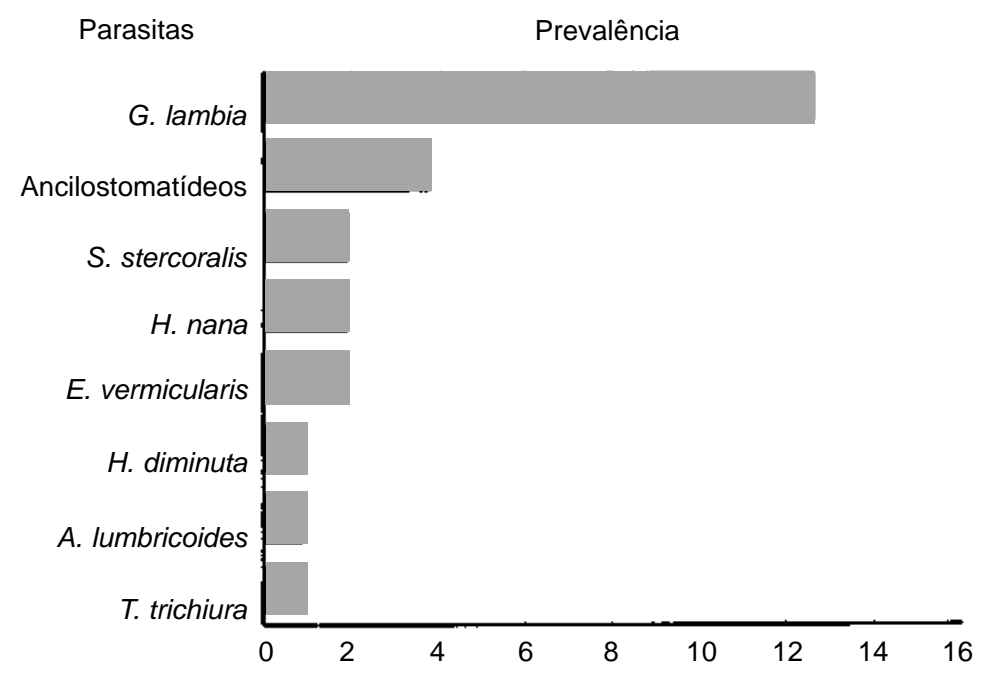

Figura 2 - Coeficientes de prevalência de parasitoses intestinais, segundo agente infeccioso, em escolares do distrito de Martinésia, Uberlândia (MG), 1995.

a espécie comensal mais freqüente, esteve presente em $8,7 \%$ dos alunos. As outras foram Iodamoeba butschlii, Endolimax nana e Eimeria sardinae $(2,9 \%, 1,9 \%$ e $1,0 \%$ respectivamente).

\section{DISCUSSÃO}

Diferentes metodologias têm sido adotadas na determinação dos índices de prevalência das parasitoses intestinais, incluindo desde a 
Ferreira CB, Marçal Junior O. Enteroparasitoses em escolares do distrito de Martinésia, Uberlândia, MG: um estudo-piloto. Revista da Sociedade Brasileira de Medicina Tropical 30:373-377, set-out, 1997.

determinação direta durante necrópsias ${ }^{2}$ até o levantamento de dados de rotina em postos de saúde9, o que dificulta consideravelmente a comparação dos resultados dos diferentes trabalhos. Na presente pesquisa, utilizou-se o método de sedimentação espontânea em função de sua eficiência e economia14, como também por ter sido o método empregado por outros autores na investigação das enteroparasitoses em Uberlândia e região1 3 .

O coeficiente geral de prevalência de enteroparasitoses $(22,3 \%)$ e a ocorrência de um único caso de poliparasitismo entre os escolares pesquisados podem ser considerados resultados alentadores, sobretudo porque o grupo estudado é um importante grupo de risco (crianças e jovens) e pelo fato de Martinésia ser uma comunidade rural. Acreditamos que este quadro seja um reflexo da forte pressão exercida pelo padrão de saneamento básico daquele distrito (incluindo água encanada e rede de esgoto) sobre os níveis de transmissão das infecções intestinais. Vale lembrar que o saneamento básico é uma das medidas que causam maior impacto sobre algumas das principais doenças humanas, incluindo ascaridíase e diarréias 810.

Não foram observadas diferenças estatisticamente significativas ( $p>0,05)$ nos coeficientes de prevalência das enteroparasitoses, segundo local de habitação, sexo e grupo etário. Do mesmo modo, quase todas as variáveis testadas para protozooses e helmintoses não apresentaram diferenças notáveis. A única exceção foi verificada na prevalência de protozooses segundo sexo, na qual o sexo feminino apresentou uma prevalência de 19,2\% contra 5,9\% do masculino ( $p<0,05)$. Acreditamos que esta diferença possa estar associada aos padrões comportamentais dos grupos pesquisados, o que será objeto de análise nas próximas etapas desta investigação em Martinésia quando estaremos determinando os fatores de risco para aquisição destas infecções.

Giardia lamblia foi o único protozoário patogênico registrado entre os escolares de Martinésia, apresentando um coeficiente de prevalência de $12,6 \%$ (Figura 2). Este resultado se coaduna com os obtidos em recentes levantamentos parasitológicos, que demonstram que a giardíase é uma das principais parasitoses intestinais entre as crianças brasileiras 135 . Quanto às helmintoses intestinais, o grupo etário em que se observou maior ocorrência foi 8-9 anos (20,2\%), enquanto no grupo etário de 12 13 anos, não houve sequer um caso de helmintose (Figura 1). Estes dados estão de acordo com Pedrazzani e cols 15 que registraram, em trabalho realizado no subdistrito de Santa Eudóxia, São Carlos, SP, uma maior positividade para helmintoses intestinais (51,9\%) no extrato de 8 a 12 anos e baixa freqüência de casos nas crianças acima de 12 anos.

Os menores coeficientes de prevalência entre os escolares de Martinésia foram registrados para as infecções por Ascaris lumbricoides e Trichuris trichiura (1,0\%) (Figura 2). Do mesmo modo, Bebert-Ferreira e cols3 registraram uma prevalência de 1,07\% para ascaridíase e nenhum caso de infecção por T. Trichiura entre pré-escolares da Escola de Educação Básica da Universidade Federal de Uberlândia. Estes valores são bastante inferiores aos índices globais de prevalência das respectivas parasitoses 14 17, sugerindo um baixo nível de transmissão. Por outro lado, devemos considerar que os baixos índices de infecção por Enterobius vermicularis, Strongyloides stercoralis e a não ocorrência de Taenia sp entre as crianças pesquisadas em Martinésia possam ter sofrido alguma influência do método de exame de fezes utilizado, já que o mesmo não é o mais adequado para identificação das referidas espécies 14 . Na seqüência deste trabalho serão realizados exames de fezes específicos envolvendo toda a comunidade, incluindo novamente os escolares, o que deverá dirimir esta questão.

\section{AGRADECIMENTOS}

Agradecemos à Maria da Graça Marçal e aos demais técnicos do Laboratório de Parasitologia da Universidade Federal de Uberlândia; à direção, professores e funcionários da Escola Municipal Martinésia e a toda comunidade do distrito de Martinésia por sua colaboração na realização deste trabalho.

\section{SUMMARY}

This work evaluates the occurence of intestinal parasites in the students of the Martinesia district, Uberlândia municipality, Minas Gerais State. A total of 103 children were examined, from september to november 1995, according to Lutz or Hoffman, Pons and Janer method. The overall prevalence rate was $22.3 \%$ and the highest indices of infection were observed in 8-9 age group (34.8\%), in village dwellers (30.0\%) and in females (26.9\%). Helminthosis 
and protozoosis showed similar prevalence rates (10.7\% and $12.6 \%$, respectively). Giardia lamblia was the unique protozoan parasite verified and only one case of poliparasitism was found. We concluded that: the prevalence of enteroparasitosis in the studied group is lower than expectations for a rural community, what is, probably, a consequence of the good sanitary conditions presenting in that district.

Key-words: Enteroparasitosis. Intestinal parasites. Prevalence.

\section{REFERÊNCIAS BIBLIOGRÁFICAS}

1. Almeida LP, Costa-Cruz JM, Incidência de enteroparasitoses em habitantes do município de Araguari, Minas Gerais. Revista do Centro de Ciências Biomédicas da Univiversidade Federal de Uberlândia 4:9-17, 1988.

2. Almeida JA, Araújo MBM, Rodrigues MLPR, Reis MA, Teixeira VPA. Prevalência de enteroparasitoses em fezes colhidas durante necropsias. Revista da Sociedade Brasileira de Medicina Tropical 24:27-29, 1991

3. Berbert-Ferreira M, Costa-Cruz JM, Moraes MMAR, Cardoso MLG, Oliveira AM. Parasitas intestinais em pré-escolares da escola de Educação Básica da Universidade Federal de Uberlândia, Minas Gerais no ano de 1989. Revista do Centro de Ciências Biomédicas da Universidade Federal de Uberlândia 6:15-19, 1990.

4. Campos R, Briques W, Belda Neto M, Souza JM, Katz N, Salata E, Dacal ARG, Dourado H, Castanho REP, Gurvitz R, Zingano A, Pereira GJM, Ferriolli Filho F, Camilo-Coura L, Faria JAS, Cimerman B, Siqueira Filho JB, Prata A. Levantamento multicêntrico de parasitoses intestinais no Brasil. Rhodia - Grupo Rhône-Poulen, 1988.

5. Cardoso GS, Santana ADC, Aguiar CP. Prevalência e aspectos epidemiológicos da giardíase em creches no município de Aracaju, SE, Brasil. Revista da Sociedade Brasileira de Medicina Tropical 28:2531, 1995.

6. Crompton DWT. The prevalence of Ascariasis. Parasitology Today 4:162-169, 1988.

7. Dean AG, Dean JA, Burton AH, Dicker RC. EPIINFO Version 5 (A Word processing database and statistic program on microcomputers. USD. INC. Stone Mountain, Georgia, 1990.

8. Esrey SA, Potash JB, Roberts L, Shiff. Effects of improved water supply and sanitatiom on ascaris diarrhoeas, dracunculiasis, hookworm infection, schistosomiasis and trachoma. Bulletin of the World Health Organization 69: 609-621, 1991.

9. Gioia I. Prevalência de parasitoses intestinais entre os usuários do Centro de Saúde do Distrito de Souzas, Campinas, SP (1986-1990). Revista da Sociedade Brasileira de Medicina Tropical 25:177182, 1992.

10. Gross R, Schell B, Molina MCB, Leão MAC, Strack U. The impact of improvement of water supply and sanitation facilities on diarrhea and intestinal parasites: a brazilian experience with children in two low-income urban communities. Revista de Saúde Pública de São Paulo 23:214-220, 1989.

11. Hoffman WA, Pons JA, Janer SL.The sedimentation concentration method in Schistosomiasis mansoni. Puerto Rico Journal of Public Health 9:283-291, 1934.

12. Lutz AO. Schistosoma mansoni e a schistosomose segundo observações feitas no Brazil. Memórias do Instituto Oswaldo Cruz 11:121-155, 1919.

13. Mello DA, Pripas, S, Fucci, M, Santoro MC, Pedrazzani ES. Helimntoses intestinais: I. Conhecimentos, atitudes e percepção da população. Revista de Saúde Pública de São Paulo 22:140-149, 1988.

14. Neves DP. Parasitologia Humana. 9a edição. São Paulo, Livraria Atheneu, 1995.

15. Pedrazzani ES, Mello DA, Pripas S, Fucci S, Barbosa CAA, Santoro MCM. Helmintoses intestinais. Revista de Saúde Pública de São Paulo 22:384389, 1988.

16. Pellon $\mathrm{AB}$, Teixeira I. Inquérito helmintológico escolar em cinco estados das regiões Leste, Sul e Centro-Oeste. Rio de Janeiro, divisão de organização Sanitária, 1953.

17. Rey L. Parasitologia médica. Editora Guanabara Koogan, Rio de Janeiro, RJ, 1992.

18. Vinha C. Incidência no Brasil de helmintos transmitidos pelo solo. Rotina cooproscópica do Ex- Departamento Nacional de Endemias Rurais. Revista Brasileira de Malariologia e Doenças Tropicais 23: 3-17, 1971.

19. Warren KS, Bowers JZ. Parasitology: a Global Perspective. Springer-Verlag, New York, 1983. 